\title{
Exact Solutions and Optical Soliton Solutions of the Nonlinear Biswas-Milovic Equation with Dual-Power Law Nonlinearity
}

\author{
E.M.E. ZAYED ${ }^{a, *}$ AND A.-G. AL-NOWEHY ${ }^{b, c}$ \\ ${ }^{a}$ Department of Mathematics, Faculty of Sciences, Zagazig University, Zagazig, Egypt \\ ${ }^{b}$ Department of Mathematics, Faculty of Education, Ain Shams University, Roxy, Hiliopolis, Cairo, Egypt \\ ${ }^{c}$ Department of Mathematics, Faculty of Education and Science, Taiz University, Taiz, Yemen
}

(Received May 9, 2016; in final form January 16, 2017)

\begin{abstract}
In this article, we apply two mathematical tools, namely the first integral method and the rational $\left(G^{\prime} / G\right)$ expansion method to construct the exact solutions with parameters of the nonlinear Biswas-Milovic equation with dual-power law nonlinearity. When these parameters take special values, the solitary wave solutions are derived from the exact solutions. We compare between the results yielding from these integration tools. A comparison between our results in this paper and the well-known results is also given.
\end{abstract}

DOI: 10.12693/APhysPolA.131.240

PACS/topics: first integral method, rational $\left(G^{\prime} / G\right)$-expansion method, Biswas-Milovic equation, solitary wave solutions, exact solutions and optical soliton solutions

\section{Introduction}

In the recent years, investigations of exact solutions to nonlinear partial differential equations (PDEs) play an important role in the study of nonlinear physical phenomena such as fluid mechanics, hydrodynamics, optics, plasma physics, solid state physics, biology and so on. Several methods for finding the exact solutions to nonlinear equations in mathematical physics have been presented, such as the inverse scattering method [1], the Hirota bilinear transform method [2], the truncated Painlevé expansion method [3-6], the Bäcklund transform method [7, 8], the exp-function method [9-11], the tanhfunction method [12, 13], the Jacobi elliptic function expansion method [14-18], the $\left(G^{\prime} / G\right)$-expansion method [19-25], the modified $\left(G^{\prime} / G\right)$-expansion method [26], the $\left(G^{\prime} / G, 1 / G\right)$-expansion method [27-30], the modified simple equation method [11,31-33], the multiple expfunction algorithm method $[34,35]$, the transformed rational function method [36], the local fractional series expansion method [37], the first integral method [38-40], the generalized Riccati equation mapping method [17, 18, $41,42]$, the soliton ansatz method [11,33, 43-54], the new ansatz method [55, 56], the complex amplitude ansatz method [57, 58], the variational method [59,60], the extended trail equation method [61], the Lie symmetry analysis [62,63], the $F$-expansion method [64], the method of undetermined coefficient [65] and so on.

The objective of this article is to use two mathematical tools, namely the first integral method and the rational $\left(G^{\prime} / G\right)$-expansion method to find the exact solutions and the solitary wave solutions of the Biswas-Milovic equation [66-69]:

*corresponding author; e-mail: eme_zayed@yahoo.com

$$
\mathrm{i}\left(q^{m}\right)_{t}+a\left(q^{m}\right)_{x x}+b F\left(|q|^{2}\right) q^{m}=0,
$$

where $q(x, t)$ is a complex valued function. The coefficients $a$ and $b$ represent the group-velocity dispersion and nonlinearity, respectively. The function $F\left(|q|^{2}\right) q$ is considered to be $j$-times continuously differentiable, e.g.

$$
F\left(|q|^{2}\right) q \in \bigcup_{m, n=1}^{\infty} C^{j}\left((-n, n) \times(-m, m) ; R^{2}\right) .
$$

The independent variables $x$ and $t$ represent the spatial and temporal variables, respectively. The nonlinearity parameter $m \geqslant 1$ makes Eq. (1.1) a generalized version of the nonlinear Schrödinger equation (NLSE). If $m=1$, then Eq. (1.1) collapses to NLSE that arises in nonlinear optics, fluid mechanics, plasma physics, and several other areas. In this paper, we consider the case

$$
F(u)=u^{n}+k u^{2 n},
$$

where the parameter $n$ indicates the power law nonlinearity, while $k$ is the coefficient of the nonlinear term. The restriction $0<n<2$ is considered to avoid soliton collapse and in particular $n \neq 2$ in order to eliminate self-focusing singularity in nonlinear optics [70]. Now, Eq. (1.1) reduces to the Biswas-Milovic equation with dual-power law nonlinearity

$$
\mathrm{i}\left(q^{m}\right)_{t}+a\left(q^{m}\right)_{x x}+b\left(|q|^{2 n}+k|q|^{4 n}\right) q^{m}=0 .
$$

Equation (1.4) has been discussed in [66] using the soliton perturbation theory, in [67] using the extended tanhfunction method, in [68] using the generalized Kudryashov method and in [69] using a special kind of $\left(G^{\prime} / G\right)$ expansion method, where its exact solutions have been presented.

This article is organized as follows. In Sect. 2, the description of the first integral method is given. In Sect. 3, the description of the rational $\left(G^{\prime} / G\right)$-expansion method is obtained. In Sect. 4, we apply these two methods to construct the exact solutions and the solitary wave solutions of Eq. (1.4). In Sect. 5, some conclusions are illustrated. 


\section{Description of the first integral method}

Feng [71] has presented this method originally, which is based on the ring theory of commutative algebra. The main steps [38-40, 71] of this method are summarized as follows:

Step 1. Consider a general PDE in the form

$F\left(u, u_{x}, u_{t}, u_{x x}, u_{t t}, u_{x t}, \ldots\right)=0$,

where $F$ is a polynomial in $u=u(x, t)$ and its partial derivatives. We use the wave transformation

$$
u(x, t)=U(\xi), \quad \xi=k x+\omega t,
$$

to reduce Eq. (2.1) to the following ordinary differential equation $(\mathrm{ODE})$ :

$$
H\left(U, U^{\prime}, U^{\prime \prime}, \ldots\right)=0,
$$

where $k, \omega$ are constants and $H$ is a polynomial in $U=$ $U(\xi)$, while its total derivatives, ${ }^{\prime}=\mathrm{d} / \mathrm{d} \xi$. form

Step 2. Assume that the solution of Eq. (2.3) has the

$$
U(\xi)=X(\xi),
$$

and introduce a new independent variable $Y=Y(\xi)$ such that

$$
Y(\xi)=X^{\prime}(\xi) .
$$

Step 3. From (2.4) and (2.5), Eq. (2.3) can be converted into a system of nonlinear ODEs

$$
X^{\prime}(\xi)=Y(\xi), \quad Y^{\prime}(\xi)=F_{1}(X(\xi), Y(\xi)),
$$

where $F_{1}$ is a polynomial in $X(\xi)$ and $Y(\xi)$.

If we can find the integrals to Eqs. (2.6), then the general solutions to Eqs. (2.6) can be found directly. However, in general, it is difficult for us to realize this even for one first integral, because for a given plane autonomous system, there is no systematic theory that can tell us how to find its first integrals, or there is no a logical way for telling us what these first integrals are. We will apply the so-called Division Theorem to obtain one first integral to Eqs. (2.6) which reduces Eq. (2.3) to a first integral ODE. Exact solutions to Eq. (2.1) are then obtained by solving this equation.

Division Theorem [72, 73]: Suppose that $P(\omega, z)$ and $Q(\omega, z)$ are polynomials in the complex domain $C[\omega, z]$; and $P(\omega, z)$ is irreducible in $C[\omega, z]$. If $Q(\omega, z)$ vanishes at all zero points of $P(\omega, z)$, then there exists a polynomial $G(\omega, z)$ in $C[\omega, z]$ such that

$$
Q(\omega, z)=P(\omega, z) G(\omega, z) \text {. }
$$

\section{Description of the rational $\left(G^{\prime} / G\right)$-expansion method}

The main steps of the rational $\left(G^{\prime} / G\right)$-expansion method used in our paper can be found in [74] which are different from that obtained in [ 19-25], describing as follows:

Step 1. We consider Eqs. (2.1)-(2.3) of Sect. 2.

Step 2. We assume that the formal solution of the ODE (2.3) can be written in the following new rational form:

$$
U(\xi)=\frac{\sum_{i=0}^{n} a_{i} Q^{i}(\xi)}{\sum_{j=0}^{m} b_{j} Q^{j}(\xi)}=\frac{A[Q(\xi)]}{B[Q(\xi)]},
$$

where $Q(\xi)=\frac{G^{\prime}(\xi)}{G(\xi)}, A[Q(\xi)]=\sum_{i=0}^{n} a_{i} Q^{i}(\xi)$ and $B[Q(\xi)]=\sum_{j=0}^{m} b_{j} Q^{j}(\xi)$. The function $G=G(\xi)$ satisfies the second order linear differential equation in the form

$$
G^{\prime \prime}+\lambda_{1} G^{\prime}+\mu_{1} G=0,
$$

where $\lambda_{1}$ and $\mu_{1}$ are constants. It is easy to see that

$$
Q^{\prime}=-\left(Q^{2}+\lambda_{1} Q+\mu_{1}\right) \text {. }
$$

Taking into consideration (3.1), we obtain

$$
\begin{aligned}
& U^{\prime}(\xi)=-\left(Q^{2}+\lambda_{1} Q+\mu_{1}\right)\left[\frac{A^{\prime} B-A B^{\prime}}{B^{2}}\right] \\
& U^{\prime \prime}(\xi)=\left(2 Q+\lambda_{1}\right)\left(Q^{2}+\lambda_{1} Q+\mu_{1}\right)\left[\frac{A^{\prime} B-A B^{\prime}}{B^{2}}\right] \\
& \quad+\left(Q^{2}+\lambda_{1} Q+\mu_{1}\right)^{2} \\
& \quad \times\left[\frac{B\left(A^{\prime \prime} B-A B^{\prime \prime}\right)-2 A^{\prime} B^{\prime} B+2 A B^{\prime 2}}{B^{3}}\right]
\end{aligned}
$$

and similar for higher order differentiation terms.

Step 3. Under the terms of the given method, we suppose that the solution of Eq. (2.3) can be written in the following form:

$$
U(\xi)=\frac{a_{0}+a_{1} Q+a_{2} Q^{2}+\ldots+a_{n} Q^{n}}{b_{0}+b_{1} Q+b_{2} Q^{2}+\ldots+b_{m} Q^{m}} .
$$

To calculate the values $m$ and $n$ in (3.6) that is the pole order for the general solution of Eq. (2.3), we progress conformably as in the classical Kudryashov method on balancing the highest order nonlinear terms and the highest order derivatives of $U(\xi)$ in Eq. (2.3) and we can determine a formula of $m$ and $n$. We can receive some values of $m$ and $n$.

Step 4. We substitute (3.1) into Eq. (2.3) to get a polynomial $R(Q)$ of $Q$ and equate all the coefficients of $Q^{i}(i=0,1,2, \ldots)$ to zero, to yield a system of algebraic equations for $a_{i}(i=0,1, \ldots, n)$ and $b_{j}(j=0,1, \ldots, m)$.

Step 5. We solve the algebraic equations obtained in Step 4 using Mathematica or Maple, to get $k, \lambda, \lambda_{1}, \mu_{1}$ and the coefficients of $a_{i}(i=0,1, \ldots, n)$ and $b_{j}(j=$ $0,1, \ldots, m)$.

Step 6. It is well known $[22]$ that the ratio $\left(G^{\prime} / G\right)$ has the following forms: if $\lambda_{1}^{2}-4 \mu_{1}>0$

$$
\frac{G^{\prime}(\xi)}{G(\xi)}=\frac{-\lambda_{1}}{2}+\frac{1}{2} \sqrt{\lambda_{1}^{2}-4 \mu_{1}}
$$

$\times\left[\frac{c_{1} \sinh \left(\frac{1}{2} \xi \sqrt{\lambda_{1}^{2}-4 \mu_{1}}\right)+c_{2} \cosh \left(\frac{1}{2} \xi \sqrt{\lambda_{1}^{2}-4 \mu_{1}}\right)}{c_{1} \cosh \left(\frac{1}{2} \xi \sqrt{\lambda_{1}^{2}-4 \mu_{1}}\right)+c_{2} \sinh \left(\frac{1}{2} \xi \sqrt{\lambda_{1}^{2}-4 \mu_{1}}\right)}\right]$,

if $\lambda_{1}^{2}-4 \mu_{1}<0$

$$
\frac{G^{\prime}(\xi)}{G(\xi)}=\frac{-\lambda_{1}}{2}+\frac{1}{2} \sqrt{4 \mu_{1}-\lambda_{1}^{2}}
$$

$\times\left[\frac{c_{1} \cos \left(\frac{1}{2} \xi \sqrt{4 \mu_{1}-\lambda_{1}^{2}}\right)-c_{2} \sin \left(\frac{1}{2} \xi \sqrt{4 \mu_{1}-\lambda_{1}^{2}}\right)}{c_{1} \sin \left(\frac{1}{2} \xi \sqrt{4 \mu_{1}-\lambda_{1}^{2}}\right)+c_{2} \cos \left(\frac{1}{2} \xi \sqrt{4 \mu_{1}-\lambda_{1}^{2}}\right)}\right]$, 
if $\lambda_{1}^{2}-4 \mu_{1}=0$

$$
\frac{G^{\prime}(\xi)}{G(\xi)}=\frac{-\lambda_{1}}{2}+\frac{c_{2}}{c_{1}+c_{2} \xi},
$$

where $c_{1}$ and $c_{2}$ are arbitrary constants.

Step 7. We substitute the values $a_{i}, b_{j}, k, \lambda, \lambda_{1}$ and $\mu_{1}$ as well as the ratios (3.7)-(3.9) into (3.1) along with (2.2), we have the hyperbolic, trigonometric and rational function solutions of Eq. (2.1).

\section{Mathematical analysis}

In order to apply the first integral method and the rational $\left(G^{\prime} / G\right)$-expansion method for finding the exact solutions of Eq. (1.4), we first suppose that the solution of Eq. (1.4) can be written in the complex form

$$
\begin{aligned}
& q(x, t)=\phi(\xi) \exp (\mathrm{i} \theta), \quad \xi=x-\lambda t, \\
& \theta=k_{1} x-\omega t,
\end{aligned}
$$

where $\lambda, k_{1}$ and $\omega$ are constants.

Substituting (4.1) into Eq. (1.4) and equating the real part and the imaginary part to zero, we get

$$
\begin{gathered}
a\left(\phi^{m}\right)^{\prime \prime}+m\left(\omega-a m k_{1}^{2}\right) \phi^{m} \\
\quad+b \phi^{2 n+m}+b k \phi^{4 n+m}=0,
\end{gathered}
$$

and

$$
\lambda=2 a m k_{1} .
$$

Balancing $\left(\phi^{m}\right)^{\prime \prime}$ with $\phi^{4 n+m}$ in (4.2), we have the balance number

$$
N=\frac{1}{2 n},
$$

which is non-integer. In this case, we take into consideration the transformation

$$
\phi(\xi)=[v(\xi)]^{\frac{1}{2 n}} .
$$

Substituting (4.5) into Eq. (4.2) we have the ODE:

$$
\begin{aligned}
& 2 a m n v v^{\prime \prime}+a m(m-2 n)\left(v^{\prime}\right)^{2}+4 m n^{2}\left(\omega-a m k_{1}^{2}\right) v^{2} \\
& +4 b n^{2} v^{3}+4 b k n^{2} v^{4}=0 .
\end{aligned}
$$

\subsection{On solving Eq. (1.4) using the first integral method}

In this subsection, we apply the first integral method [73] to solve Eq. (1.4). To this aim, we set

$$
v(\xi)=X(\xi), \quad v^{\prime}(\xi)=\frac{\mathrm{d} X}{\mathrm{~d} \xi}=Y(\xi),
$$

and hence Eq. (4.6) reduces to

$$
\begin{aligned}
Y^{\prime} & =-\frac{(m-2 n)}{2 n X} Y^{2}-\frac{2 n\left(\omega-a m k_{1}^{2}\right)}{a} X-\frac{2 b n}{a m} X^{2} \\
& -\frac{2 b k n}{a m} X^{3}, \quad m \neq 2 n .
\end{aligned}
$$

Set $X=\frac{\mathrm{d} \xi}{\mathrm{d} \tau}$, where $\tau$ is a new variable, then we have

$$
\begin{gathered}
Y=\frac{\mathrm{d} X}{\mathrm{~d} \xi}=\frac{\mathrm{d} X}{\mathrm{~d} \tau} \frac{\mathrm{d} \tau}{\mathrm{d} \xi}=\frac{1}{X} \frac{\mathrm{d} X}{\mathrm{~d} \tau}, \\
Y^{\prime}=\frac{\mathrm{d} Y}{\mathrm{~d} \xi}=\frac{\mathrm{d} Y}{\mathrm{~d} \tau} \frac{\mathrm{d} \tau}{\mathrm{d} \xi}=\frac{1}{X} \frac{\mathrm{d} Y}{\mathrm{~d} \tau} .
\end{gathered}
$$

Now, we deduce that

$$
\frac{\mathrm{d} X}{\mathrm{~d} \tau}=X Y,
$$

and Eq. (4.1.2) becomes

$$
\begin{aligned}
& \frac{\mathrm{d} Y}{\mathrm{~d} \tau}=-\frac{(m-2 n)}{2 n} Y^{2}-\frac{2 n\left(\omega-a m k_{1}^{2}\right)}{a} X^{2}-\frac{2 b n}{a m} X^{3} \\
& \quad-\frac{2 b k n}{a m} X^{4} .
\end{aligned}
$$

According to the first integral method, we assume that $X(\tau)$ and $Y(\tau)$ are nontrivial solutions of (4.1.6), and $Q(X, Y)=\sum_{i=0}^{M} a_{i}(X) Y^{i}$ is an irreducible polynomial in the complex domain $C[X, Y]$ such that

$$
Q(X, Y)=\sum_{i=0}^{M} a_{i}(X) Y^{i}=0,
$$

where $a_{i}(X)(i=0,1, \ldots, M)$ are polynomials in $X(\tau)$ and $a_{i}(X) \neq 0$. Due to the Division Theorem, there exists a polynomial $h(X)+g(X) Y$, in the complex domain $C[X, Y]$ such that

$$
\begin{aligned}
& \frac{\mathrm{d} Q}{\mathrm{~d} \tau}=\frac{\mathrm{d} Q}{\mathrm{~d} X} \frac{\mathrm{d} X}{\mathrm{~d} \tau}+\frac{\mathrm{d} Q}{\mathrm{~d} Y} \frac{\mathrm{d} Y}{\mathrm{~d} \tau}= \\
& {[h(X)+g(X) Y] \sum_{i=0}^{M} a_{i}(X) Y^{i} .}
\end{aligned}
$$

Let us now discuss the following cases.

Case 1. $M=1$.

In this case, we deduce that

$$
Q(X, Y)=a_{0}(X)+a_{1}(X) Y=0,
$$

and

$$
\begin{aligned}
& {\left[\frac{\mathrm{d}}{\mathrm{d} X} a_{0}(X)+Y \frac{\mathrm{d}}{\mathrm{d} X} a_{1}(X)\right] X Y} \\
& \quad+a_{1}(X)\left[-\frac{(m-2 n)}{2 n} Y^{2}-\frac{2 n\left(\omega-a m k_{1}^{2}\right)}{a} X^{2}\right. \\
& \left.\quad-\frac{2 b n}{a m} X^{3}-\frac{2 b k n}{a m} X^{4}\right]= \\
& \quad[h(X)+g(X) Y]\left[a_{0}(X)+a_{1}(X) Y\right] .
\end{aligned}
$$

Equating the coefficients of $Y^{i}(i=2,1,0)$ on both sides of (4.1.10), we get

$$
\begin{aligned}
Y^{2} & : X \frac{\mathrm{d}}{\mathrm{d} X} a_{1}(X)-\frac{(m-2 n)}{2 n} a_{1}(X)= \\
& g(X) a_{1}(X), \\
Y^{1} & : X \frac{\mathrm{d}}{\mathrm{d} X} a_{0}(X)= \\
& h(X) a_{1}(X)+g(X) a_{0}(X), \\
Y^{0} & :-a_{1}(X)\left[\frac{2 n\left(\omega-a m k_{1}^{2}\right)}{a} X^{2}+\frac{2 b n}{a m} X^{3}+\frac{2 b k n}{a m} X^{4}\right] \\
& =h(X) a_{0}(X) .
\end{aligned}
$$

From (4.1.11), we have

$$
a_{1}(X)=c_{1} \exp \left(\int \frac{1}{X}\left[g(X)+\frac{(m-2 n)}{2 n}\right] \mathrm{d} X\right),
$$

where $c_{1}$ is a constant. Choose 


$$
g(X)=1-\frac{m}{2 n}, \quad m \neq 2 n,
$$

then $a_{1}(X)$ is a constant. For simplicity, we take $a_{1}(X)=1$. Balancing the degrees of $h(X)$ and $a_{0}(X)$, we conclude that $\operatorname{deg}(h(X))=\operatorname{deg}\left(a_{0}(X)\right)=2$ only. Suppose that

$$
\begin{aligned}
& h(X)=A_{0}+A_{1} X+A_{2} X^{2}, \\
& a_{0}(X)=B_{0}+B_{1} X+B_{2} X^{2},
\end{aligned}
$$

where $A_{i}, B_{i}(i=0,1,2)$ are arbitrary constants, such that $A_{2} \neq 0$ and $B_{2} \neq 0$.

Substituting (4.1.16) and (4.1.17) into (4.1.12) and equating the coefficients of powers of $X(\tau)$ on both sides of Eq. (4.1.12), we obtain

$$
\begin{aligned}
& X^{2}: A_{2}=B_{2}\left(\frac{m+2 n}{2 n}\right), \\
& X^{1}: A_{1}=B_{1}\left(\frac{m}{2 n}\right), \\
& X^{0}: A_{0}=B_{0}\left(\frac{m-2 n}{2 n}\right) .
\end{aligned}
$$

Consequently, (4.1.16) becomes

$$
\begin{aligned}
& h(X)=B_{0}\left(\frac{m-2 n}{2 n}\right)+B_{1}\left(\frac{m}{2 n}\right) X \\
& +B_{2}\left(\frac{m+2 n}{2 n}\right) X^{2} .
\end{aligned}
$$

Substituting (4.1.17) and (4.1.18) into (4.1.13) and equating the coefficients of powers of $X(\tau)$ on both sides of Eq. (4.1.13), we obtain a system of algebraic equations

$$
\begin{aligned}
& X^{4}:-\frac{2 b k n}{a m}=B_{2}^{2}\left(\frac{m+2 n}{2 n}\right), \\
& X^{3}:-\frac{2 b n}{a m}=B_{1} B_{2}\left(\frac{m+n}{n}\right), \\
& X^{2}:-\frac{2 n\left(\omega-a m k_{1}^{2}\right)}{a}=B_{0} B_{2}\left(\frac{m}{n}\right)+B_{1}^{2}\left(\frac{m}{2 n}\right), \\
& X^{1}: B_{0} B_{1}\left(\frac{m-n}{n}\right)=0, \\
& X^{0}: B_{0}^{2}\left(\frac{m-2 n}{2 n}\right)=0 .
\end{aligned}
$$

Since $m \neq 2 n$, and by solving algebraic equations (4.1.19), we get the results

$$
\begin{aligned}
& B_{0}=0, \quad B_{1}= \pm \sqrt{\frac{-n^{2} b(m+2 n)}{a m k(m+n)^{2}}}, \\
& B_{2}= \pm \sqrt{\frac{-4 n^{2} b k}{a m(m+2 n)}}, \quad \omega=a m k_{1}^{2}+\frac{b(m+2 n)}{4 k(m+n)^{2}},
\end{aligned}
$$

provided that $a b k<0$.

Now, (4.1.17) reduces to

$$
a_{0}(X)= \pm \sqrt{\frac{-n^{2} b(m+2 n)}{a m k(m+n)^{2}}} X
$$

$$
\pm \sqrt{\frac{-4 n^{2} b k}{a m(m+2 n)}} X^{2} .
$$

From (4.1.1), (4.1.9) and (4.1.21), we deduce that

$$
\begin{gathered}
v^{\prime}(\xi)=\mp \sqrt{\frac{-n^{2} b(m+2 n)}{a m k(m+n)^{2}}} v(\xi) \\
\mp \sqrt{\frac{-4 n^{2} b k}{a m(m+2 n)}} v^{2}(\xi) .
\end{gathered}
$$

In order to solve Eq. (4.1.22), we refer to the Bernoulli equation

$$
v^{\prime}(\xi)=l_{1} v(\xi)+l_{2} v^{\beta}(\xi),
$$

where $l_{1}, l_{2}, \beta \in \mathbb{R}, l_{1} l_{2} \neq 0, \beta \neq 1$. Its general solution is well-known [75] and has the form

$$
\begin{aligned}
& v(\xi)=\left[\frac{-l_{1} / l_{2}}{\xi_{0} \exp \left(l_{1}(1-\beta) \xi\right)+1}\right]^{\frac{1}{\beta-1}} \\
& =\left\{\begin{array}{l}
\left\{\frac{-l_{1}}{2 l_{2}}\left[1+\tanh \left(\frac{l_{1}(1-\beta)}{2} \xi-\frac{\ln \xi_{0}}{2}\right)\right]\right\}^{\frac{1}{\beta-1}}, \\
\text { if } \xi_{0}>0, \\
\left\{\frac{-l_{1}}{2 l_{2}}\left[1+\operatorname{coth}\left(\frac{l_{1}(1-\beta)}{2} \xi-\frac{\ln \left(-\xi_{0}\right)}{2}\right)\right]\right\}^{\frac{1}{\beta-1}}, \\
\text { if } \xi_{0}<0, \quad \text { if } \xi_{0}=0 .
\end{array}\right.
\end{aligned}
$$

If we choose $l_{1}=\mp \sqrt{\frac{-n^{2} b(m+2 n)}{a m k(m+n)^{2}}}, \quad l_{2}=\mp \sqrt{\frac{-4 n^{2} b k}{a m(m+2 n)}}$ and $\beta=2$, then the solution $v(\xi)$ of Eq. (4.1.22) can be found. From (4.1) and (4.5), we obtain the solitary wave solutions of Eq. (1.4) as follows: if $\xi_{0}>0$

$$
\begin{gathered}
q(x, t)=\left\{\frac{-(m+2 n)}{4 k(m+n)}[1 \pm\right. \\
\left.\left.\tanh \left(\frac{1}{2} \sqrt{\frac{-n^{2} b(m+2 n)}{a m k(m+n)^{2}}}\left(x-2 a m k_{1} t\right)-\frac{\ln \xi_{0}}{2}\right)\right]\right\}^{\frac{1}{2 n}} \\
\quad \times \exp \left(\mathrm{i}\left[k_{1} x-\left(a m k_{1}^{2}+\frac{b(m+2 n)}{4 k(m+n)^{2}}\right) t\right]\right),
\end{gathered}
$$

if $\xi_{0}<0$

$$
\begin{gathered}
q(x, t)=\left\{\frac{-(m+2 n)}{4 k(m+n)}[1 \pm\right. \\
\left.\left.\operatorname{coth}\left(\frac{1}{2} \sqrt{\frac{-n^{2} b(m+2 n)}{a m k(m+n)^{2}}}\left(x-2 a m k_{1} t\right)-\frac{\ln \left(-\xi_{0}\right)}{2}\right)\right]\right\}^{\frac{1}{2 n}} \\
\quad \times \exp \left(\mathrm{i}\left[k_{1} x-\left(a m k_{1}^{2}+\frac{b(m+2 n)}{4 k(m+n)^{2}}\right) t\right]\right\},
\end{gathered}
$$

if $\xi_{0}=0$

$$
q(x, t)=\left\{\frac{-(m+2 n)}{2 k(m+n)}\right\}^{\frac{1}{2 n}}
$$




$$
\times \exp \left(\mathrm{i}\left[k_{1} x-\left(a m k_{1}^{2}+\frac{b(m+2 n)}{4 k(m+n)^{2}}\right) t\right]\right) .
$$

The constraint conditions for the existence of the soliton solutions (4.1.25)-(4.1.27) are $k<0$ and $a b>0$.

Note that the solutions (4.1.25) and (4.1.26) are equivalent to that obtained in $[67,68]$ using different methods, but the solution (4.1.27) is new.

Case 2. $M=2$.

In this case, we deduce from (4.1.7) and (4.1.8) that $Q(X, Y)=a_{0}(X)+a_{1}(X) Y+a_{2}(X) Y^{2}=0$,

and

$$
\begin{aligned}
& {\left[\frac{\mathrm{d}}{\mathrm{d} X} a_{0}(X)+Y \frac{\mathrm{d}}{\mathrm{d} X} a_{1}(X)+Y^{2} \frac{\mathrm{d}}{\mathrm{d} X} a_{2}(X)\right] X Y} \\
& \quad+\left[a_{1}(X)+2 a_{2}(X) Y\right]\left[-\frac{(m-2 n)}{2 n} Y^{2}\right. \\
& \left.\quad-\frac{2 n\left(\omega-a m k_{1}^{2}\right)}{a} X^{2}-\frac{2 b n}{a m} X^{3}-\frac{2 b k n}{a m} X^{4}\right]= \\
& \quad[h(X)+g(X) Y]\left[a_{0}(X)+a_{1}(X) Y+a_{2}(X) Y^{2}\right] .
\end{aligned}
$$

Equating the coefficients of $Y^{i}(i=3,2,1,0)$ on both sides of (4.1.29), we have

$$
\begin{aligned}
Y^{3} & : X \frac{\mathrm{d}}{\mathrm{d} X} a_{2}(X)-\frac{(m-2 n)}{n} a_{2}(X)=g(X) a_{2}(X), \\
Y^{2} & : X \frac{\mathrm{d}}{\mathrm{d} X} a_{1}(X)-\frac{(m-2 n)}{2 n} a_{1}(X)=h(X) a_{2}(X) \\
& +g(X) a_{1}(X), \\
Y^{1} & : X \frac{\mathrm{d}}{\mathrm{d} X} a_{0}(X)+2 a_{2}(X) \\
\quad \times & {\left[-\frac{2 n\left(\omega-a m k_{1}^{2}\right)}{a} X^{2}-\frac{2 b n}{a m} X^{3}-\frac{2 b k n}{a m} X^{4}\right]=} \\
\quad h(X) a_{1}(X)+g(X) a_{0}(X), & (4.1 .32) \\
Y^{0} & :-a_{1}(X)\left[\frac{2 n\left(\omega-a m k_{1}^{2}\right)}{a} X^{2}+\frac{2 b n}{a m} X^{3}+\frac{2 b k n}{a m} X^{4}\right] \\
& =h(X) a_{0}(X) .
\end{aligned}
$$

From (4.1.30), we have

$$
a_{2}(X)=c_{2} \exp \left(\int \frac{1}{X}\left[g(X)+\frac{(m-2 n)}{n}\right] \mathrm{d} X\right),
$$

where $c_{2}$ is a constant. If we choose

$$
g(X)=2-\frac{m}{n}, \quad m \neq 2 n,
$$

then $a_{2}(X)$ is a constant. For simplicity, we take $a_{2}(X)=1$. Balancing the degrees of $a_{0}(X), a_{1}(X)$ and $h(X)$, we conclude that $\operatorname{deg}(h(X))=\operatorname{deg}\left(a_{1}(X)\right)=2$, and $\operatorname{deg}\left(a_{0}(X)\right)=4$ only. Therefore, we assume that

$$
\begin{aligned}
& h(X)=C_{0}+C_{1} X+C_{2} X^{2}, \\
& a_{1}(X)=B_{0}+B_{1} X+B_{2} X^{2}, \\
& a_{0}(X)=A_{0}+A_{1} X+A_{2} X^{2}+A_{3} X^{3} \\
& \quad+A_{4} X^{4},
\end{aligned}
$$

where $C_{i}, B_{i}(i=0,1,2)$ and $A_{j}(j=0,1, \ldots, 4)$ are arbitrary constants, such that $C_{2} \neq 0, B_{2}$ and $A_{4} \neq 0$.

Now, (4.1.31) reduces to

$$
\begin{aligned}
& \left(\frac{m}{2 n}-1\right) B_{0}+\frac{m}{2 n} B_{1} X+\left(1+\frac{m}{2 n}\right) B_{2} X^{2}= \\
& C_{0}+C_{1} X+C_{2} X^{2} .
\end{aligned}
$$

Equating the coefficients of powers of $X(\tau)$ on both sides of Eq. (4.1.39), we obtain

$$
\begin{aligned}
& X^{2}: C_{2}=B_{2}\left(\frac{m+2 n}{2 n}\right), \\
& X^{1}: C_{1}=B_{1}\left(\frac{m}{2 n}\right), \\
& X^{0}: C_{0}=B_{0}\left(\frac{m-2 n}{2 n}\right) .
\end{aligned}
$$

Consequently, (4.1.36) becomes

$$
\begin{aligned}
& h(X)=B_{0}\left(\frac{m-2 n}{2 n}\right)+B_{1}\left(\frac{m}{2 n}\right) X \\
& \quad+B_{2}\left(\frac{m+2 n}{2 n}\right) X^{2} .
\end{aligned}
$$

Also, (4.1.32) reduces to

$$
\begin{aligned}
& \left(\frac{m}{n}-2\right) A_{0}+\left(\frac{m}{n}-1\right) A_{1} X \\
& +\left[\frac{m}{n} A_{2}-\frac{4 n\left(\omega-a m k_{1}^{2}\right)}{a}\right] X^{2} \\
& +\left[\left(\frac{m}{n}+1\right) A_{3}-\frac{4 b n}{a m}\right] X^{3} \\
& +\left[\left(\frac{m}{n}+2\right) A_{4}-\frac{4 b k n}{a m}\right] X^{4}= \\
& \quad B_{0}^{2}\left(\frac{m-2 n}{2 n}\right)+B_{0} B_{1}\left(\frac{m-n}{n}\right) X \\
& +\frac{m}{2 n}\left[B_{1}^{2}+2 B_{0} B_{2}\right] X^{2}+B_{1} B_{2}\left(\frac{m+n}{n}\right) X^{3} \\
& +B_{2}^{2}\left(\frac{m+2 n}{2 n}\right) X^{4} .
\end{aligned}
$$

Equating the coefficients of powers of $X(\tau)$ on both sides of Eq. (4.1.41), we have

$$
\begin{aligned}
& X^{4}: A_{4}=\frac{1}{2} B_{2}^{2}+\frac{4 b k n^{2}}{a m(m+2 n)}, \\
& X^{3}: A_{3}=B_{1} B_{2}+\frac{4 b n^{2}}{a m(m+n)}, \\
& X^{2}: A_{2}=\frac{1}{2} B_{1}^{2}+B_{0} B_{2}+\frac{4 n^{2}\left(\omega-a m k_{1}^{2}\right)}{a m},
\end{aligned}
$$




$$
\begin{aligned}
& X^{1}: A_{1}=B_{0} B_{1}, \\
& X^{0}: A_{0}=\frac{1}{2} B_{0}^{2} .
\end{aligned}
$$

Consequently, (4.1.38) becomes

$$
\begin{aligned}
& a_{0}(X)=\frac{1}{2} B_{0}^{2}+B_{0} B_{1} X \\
& +\left[\frac{1}{2} B_{1}^{2}+B_{0} B_{2}+\frac{4 n^{2}\left(\omega-a m k_{1}^{2}\right)}{a m}\right] X^{2} \\
& +\left[B_{1} B_{2}+\frac{4 b n^{2}}{a m(m+n)}\right] X^{3} \\
& +\left[\frac{1}{2} B_{2}^{2}+\frac{4 b k n^{2}}{a m(m+2 n)}\right] X^{4} .
\end{aligned}
$$

Substituting (4.1.37), (4.1.40), and (4.1.42) into (4.1.33) and equating the coefficients of powers of $X(\tau)$ on both sides of Eq. (4.1.13), we obtain a system of algebraic equations, which can be solved to get the results

$$
\begin{aligned}
& B_{0}=0, \quad B_{1}= \pm \sqrt{\frac{-4 n^{2} b(m+2 n)}{a m k(m+n)^{2}}} \\
& B_{2}= \pm \sqrt{\frac{-16 b k n^{2}}{a m(m+2 n)}}, \quad \omega=a m k_{1}^{2}+\frac{b(m+2 n)}{4 k(m+n)^{2}}
\end{aligned}
$$

provided that $a b k<0$.
Now, (4.1.42) becomes

$$
\begin{aligned}
& a_{0}(X)=-\frac{n^{2} b(m+2 n)}{a m k(m+n)^{2}} X^{2}-\frac{4 b n^{2}}{a m(m+n)} X^{3} \\
& -\frac{4 b k n^{2}}{a m(m+2 n)} X^{4} .
\end{aligned}
$$

On solving Eq. (3.1.24), we have

$$
Y=-\frac{1}{2} a_{1} \pm \frac{1}{2} \sqrt{a_{1}^{2}-4 a_{0}} .
$$

It is easy to show that $a_{1}^{2}-4 a_{0}=0$, and consequently, we have

$$
Y=\mp \sqrt{\frac{-n^{2} b(m+2 n)}{a m k(m+n)^{2}}} X \mp \sqrt{\frac{-4 b k n^{2}}{a m(m+2 n)}} X^{2} .
$$

From (4.1.1) and (4.1.46), we deduce that

$$
v^{\prime}(\xi)=\mp \sqrt{\frac{-n^{2} b(m+2 n)}{a m k(m+n)^{2}}} v(\xi) \mp \sqrt{\frac{-4 b k n^{2}}{a m(m+2 n)}} v^{2}(\xi),
$$

which is the same form as (4.1.22) obtained when $M=1$. Therefore, we have the same solutions (4.1.25)-(4.1.27). Finally, we note that the two cases $M=1$ and $M=2$ give the same exact solutions of Eq. (1.4). For the case $M \geqslant 3$, the discussions become more complicated, which are omitted here and are left as an open problem for the researchers.

\subsection{On solving Eq. (1.4) using the rational $\left(G^{\prime} / G\right)$-expansion method}

In this subsection, we solve Eq. (1.4) using the rational $\left(G^{\prime} / G\right)$-expansion method. To this aim, balancing $v v^{\prime \prime}$ and $v^{4}$ in (4.6), then the following relation is attained:

$(n-m)+(n-m)+2=4(n-m) \Longrightarrow n=m+1$.

If we choose $m=1$ and $n=2$, then the formal solution of Eq. (4.6) has the form

$$
v(\xi)=\frac{a_{0}+a_{1} Q+a_{2} Q^{2}}{b_{0}+b_{1} Q},
$$

and consequently,

$$
\begin{aligned}
& v^{\prime}(\xi)=-\left(Q^{2}+\lambda_{1} Q+\mu_{1}\right)\left[\frac{\left(a_{1}+2 a_{2} Q\right)\left(b_{0}+b_{1} Q\right)-b_{1}\left(a_{0}+a_{1} Q+a_{2} Q^{2}\right)}{\left(b_{0}+b_{1} Q\right)^{2}}\right], \\
& v^{\prime \prime}(\xi)=\left(2 Q+\lambda_{1}\right)\left(Q^{2}+\lambda_{1} Q+\mu_{1}\right)\left[\frac{\left(a_{1}+2 a_{2} Q\right)\left(b_{0}+b_{1} Q\right)-b_{1}\left(a_{0}+a_{1} Q+a_{2} Q^{2}\right)}{\left(b_{0}+b_{1} Q\right)^{2}}\right] \\
& +\left(Q^{2}+\lambda_{1} Q+\mu_{1}\right)^{2}\left\{\frac{2\left(b_{0}+b_{1} Q\right)\left[a_{2}\left(b_{0}+b_{1} Q\right)-b_{1}\left(a_{1}+2 a_{2}\right)\right]+2 b_{1}^{2}\left(a_{0}+a_{1} Q+a_{2} Q^{2}\right)}{\left(b_{0}+b_{1} Q\right)^{3}}\right\} .
\end{aligned}
$$

Substituting (4.2.2)-(4.2.4) into (4.6), collecting the coefficients of each power of $Q^{i},(i=0,1, \ldots, 8)$ and setting each of the coefficients to zero, we get a system of algebraic equations, which can be solved using the Maple, to get the following sets:

Set 1

$$
a_{0}=0, \quad b_{0}=0, \quad a_{2}=\frac{b_{1}}{2 n} \sqrt{\frac{-a m(m+2 n)}{k b}}, \quad \lambda_{1}=\frac{n}{\sqrt{\frac{-a m(m+2 n)}{k b}}}\left[\frac{4 a_{1}}{b_{1}}+\frac{(m+2 n)}{k(m+n)}\right], \quad a_{1}=a_{1},
$$




$$
\mu_{1}=-2 a_{1} b n^{2}\left[\frac{b_{1}}{a m b_{1}^{2}(m+n)}+\frac{2 a_{1} k}{a m b_{1}^{2}(m+2 n)}\right], \quad \omega=a m k_{1}^{2}+\frac{b(m+2 n)}{4 k(m+n)^{2}}, b_{1}=b_{1},
$$

provided that $a b k<0$.

Substituting (4.2.5) into (4.2.2) yields

$$
v(\xi)=\frac{a_{1}}{b_{1}}+\frac{1}{2 n} \sqrt{\frac{-a m(m+2 n)}{k b}}\left(\frac{G^{\prime}(\xi)}{G(\xi)}\right) .
$$

From (4.5),(4.1),(3.7) and using (4.2.6), we have the hyperbolic solution of Eq. (1.4):

$$
\begin{aligned}
& q(x, t)=\left[\frac{-(m+2 n)}{4 k(m+n)}\left(1 \pm \frac{c_{1} \sinh \left(\frac{1}{2} \sqrt{\lambda_{1}^{2}-4 \mu_{1}} \xi\right)+c_{2} \cosh \left(\frac{1}{2} \sqrt{\lambda_{1}^{2}-4 \mu_{1}} \xi\right)}{c_{1} \cosh \left(\frac{1}{2} \sqrt{\lambda_{1}^{2}-4 \mu_{1}} \xi\right)+c_{2} \sinh \left(\frac{1}{2} \sqrt{\lambda_{1}^{2}-4 \mu_{1}} \xi\right)}\right)\right]^{\frac{1}{2 n}} \\
& \quad \times \exp \left(\mathrm{i}\left[k_{1} x-\left(a m k_{1}^{2}+\frac{b(m+2 n)}{4 k(m+n)^{2}}\right) t\right]\right),
\end{aligned}
$$

where $\xi=x-2 a m k_{1} t$ and $\lambda_{1}^{2}-4 \mu_{1}=\frac{-n^{2} b(m+2 n)}{a m k(m+n)^{2}}>0$, provided that $a b>0$ and $k<0$.

If $c_{2}=0, c_{1} \neq 0$, then we have the dark soliton solution of Eq. (1.4):

$$
q(x, t)=\left[\frac{-(m+2 n)}{4 k(m+n)}\left(1 \pm \tanh \left(\frac{1}{2} \sqrt{\frac{-n^{2} b(m+2 n)}{a m k(m+n)^{2}} \xi}\right)\right)\right]^{\frac{1}{2 n}} \exp \left(\mathrm{i}\left[k_{1} x-\left(a m k_{1}^{2}+\frac{b(m+2 n)}{4 k(m+n)^{2}}\right) t\right]\right),
$$

which is equivalent to the dark soliton solution (4.1.25).

If $c_{1}=0, c_{2} \neq 0$, then we have the singular soliton solution of Eq. (1.4):

$$
q(x, t)=\left[\frac{-(m+2 n)}{4 k(m+n)}\left(1 \pm \operatorname{coth}\left(\frac{1}{2} \sqrt{\frac{-n^{2} b(m+2 n)}{a m k(m+n)^{2}} \xi}\right)\right)\right]^{\frac{1}{2 n}} \exp \left(\mathrm{i}\left[k_{1} x-\left(a m k_{1}^{2}+\frac{b(m+2 n)}{4 k(m+n)^{2}}\right) t\right]\right)
$$

which is equivalent to the singular soliton solution (4.1.26).

If $c_{1}=c_{2} \neq 0$, then we have the rational solution of Eq. (1.4):

$$
q(x, t)=\left[\frac{-(m+2 n)}{2 k(m+n)}\right]^{\frac{1}{2 n}} \exp \left(\mathrm{i}\left[k_{1} x-\left(a m k_{1}^{2}+\frac{b(m+2 n)}{4 k(m+n)^{2}}\right) t\right]\right),
$$

which is equivalent to the rational solution (4.1.27).

The constraint conditions for the existence of the soliton solutions (4.2.8)-(4.2.10) are $k<0$ and $a b>0$.

Set 2

$$
\begin{aligned}
& a_{0}=\sqrt{\frac{-b n^{2}(m+2 n)}{a m k(m+n)^{2}}}\left[a_{1}+\frac{2 a_{1}^{2} k(m+n)}{b_{1}(m+2 n)}\right], \quad \lambda_{1}=\sqrt{\frac{-b n^{2}(m+2 n)}{a m k(m+n)^{2}}}\left[1+\frac{4 a_{1} k(m+n)}{b_{1}(m+2 n)}\right], \quad b_{0}=0, \quad a_{2}=0, \\
& \mu_{1}=\frac{-2 a_{1} b n^{2}}{a m b_{1}}\left[\frac{1}{(m+n)}+\frac{2 a_{1} k}{b_{1}(m+2 n)}\right], \quad \omega=a m k_{1}^{2}+\frac{b(m+2 n)}{4 k(m+n)^{2}}, \quad a_{1}=a_{1}, \quad b_{1}=b_{1},
\end{aligned}
$$

provided that $a b k<0$.

Substituting (4.2.11) into (4.2.2) yields

$$
v(\xi)=\frac{a_{1}}{b_{1}}\left\{1+\sqrt{\frac{-b n^{2}(m+2 n)}{a m k(m+n)^{2}}}\left[1+\frac{2 a_{1} k(m+n)}{b_{1}(m+2 n)}\right] /\left(\frac{G^{\prime}(\xi)}{G(\xi)}\right)\right\} .
$$

From (4.5),(4.1),(3.7) and using (4.2.12), we have the hyperbolic solution of Eq. (1.4):

$$
\begin{aligned}
& q(x, t)=\left(\frac{a_{1}}{b_{1}}\left\{1-\frac{2\left[b_{1}(m+2 n)+2 a_{1} k(m+n)\right]}{\left.4 a_{1} k(m+n)+b_{1}(m+2 n)\left(1 \mp \frac{c_{1} \sinh \left(\frac{1}{2} \sqrt{\lambda_{1}^{2}-4 \mu_{1}} \xi\right)+c_{2} \cosh \left(\frac{1}{2} \sqrt{\lambda_{1}^{2}-4 \mu_{1}} \xi\right)}{c_{1} \cosh \left(\frac{1}{2} \sqrt{\lambda_{1}^{2}-4 \mu_{1}} \xi\right)+c_{2} \sinh \left(\frac{1}{2} \sqrt{\lambda_{1}^{2}-4 \mu_{1}} \xi\right)}\right\}\right)}\right)\right)^{\frac{1}{2 n}} \\
& \quad \times \exp \left(\mathrm{i}\left[k_{1} x-\left(a m k_{1}^{2}+\frac{b(m+2 n)}{4 k(m+n)^{2}}\right) t\right]\right) .
\end{aligned}
$$

where $\lambda_{1}^{2}-4 \mu_{1}=\frac{-n^{2} b(m+2 n)}{a m k(m+n)^{2}}>0$, provided that $a_{1} b_{1}>0$ and $a b k<0$.

If $c_{2}=0, c_{1} \neq 0$, then we have the dark soliton solution of Eq. (1.4): 


$$
\begin{aligned}
& q(x, t)=\left(\frac{a_{1}}{b_{1}}\left\{1-\frac{2\left[b_{1}(m+2 n)+2 a_{1} k(m+n)\right]}{4 a_{1} k(m+n)+b_{1}(m+2 n)\left(1 \mp \tanh \left(\frac{1}{2} \sqrt{\frac{-n^{2} b(m+2 n)}{a m k(m+n)^{2}}} \xi\right)\right)}\right\}\right)^{\frac{1}{2 n}} \\
& \quad \times \exp \left(\mathrm{i}\left[k_{1} x-\left(a m k_{1}^{2}+\frac{b(m+2 n)}{4 k(m+n)^{2}}\right) t\right]\right) .
\end{aligned}
$$

If $c_{1}=0, c_{2} \neq 0$, then we have the singular soliton solution of Eq. (1.4):

$$
\begin{aligned}
& q(x, t)=\left(\frac{a_{1}}{b_{1}}\left\{1-\frac{2\left[b_{1}(m+2 n)+2 a_{1} k(m+n)\right]}{4 a_{1} k(m+n)+b_{1}(m+2 n)\left[1 \mp \operatorname{coth}\left(\frac{1}{2} \sqrt{\frac{-n^{2} b(m+2 n)}{a m k(m+n)^{2}}} \xi\right)\right]}\right\}\right)^{\frac{1}{2 n}} \\
& \quad \times \exp \left(\mathrm{i}\left[k_{1} x-\left(a m k_{1}^{2}+\frac{b(m+2 n)}{4 k(m+n)^{2}}\right) t\right]\right) .
\end{aligned}
$$

If $c_{1}=c_{2} \neq 0$, then we have the same rational solution (4.2.10).

The constraint conditions for the existence of the soliton solutions (4.2.14) and (4.2.15) are $a_{1} b_{1}>0$ and $a b k<0$.

\section{Set 3}

$$
\begin{aligned}
& a_{0}=0, \quad a_{1}=\left[\mp \frac{a m b_{0}}{2 n b} \sqrt{\frac{-b(m+2 n)}{a m k}}-\frac{b_{1}(m+2 n)}{2 k(m+n)}\right], \quad \lambda_{1}= \pm \sqrt{\frac{-b n^{2}(m+2 n)}{a m k(m+n)^{2}}}, \\
& b_{0}=b_{0}, \quad a_{2}=0, \quad \mu_{1}=0, \quad \omega=a m k_{1}^{2}+\frac{b(m+2 n)}{4 k(m+n)^{2}}, \quad b_{1}=b_{1},
\end{aligned}
$$

provided that $a b k<0$.

Substituting (4.2.16) into (4.2.2) yields

$$
v(\xi)=\frac{\left[\mp \frac{a m b_{0}}{2 n b} \sqrt{\frac{-b(m+2 n)}{a m k}}-\frac{b_{1}(m+2 n)}{2 k(m+n)}\right]\left(\frac{G^{\prime}(\xi)}{G(\xi)}\right)}{b_{0}+b_{1}\left(\frac{G^{\prime}(\xi)}{G(\xi)}\right)} .
$$

From (4.5),(4.1),(3.7) and using (4.2.17), we have the hyperbolic solution of Eq. (1.4):

$$
\begin{aligned}
& q(x, t)=\left\{\frac{\left[ \pm \frac{a m b_{0}}{2 n b} \sqrt{\frac{-b(m+2 n)}{a m k}}+\frac{b_{1}(m+2 n)}{2 k(m+n)}\right]\left[1-\frac{c_{1} \sinh \left(\frac{1}{2} \lambda_{1} \xi\right)+c_{2} \cosh \left(\frac{1}{2} \lambda_{1} \xi\right)}{c_{1} \cosh \left(\frac{1}{2} \lambda_{1} \xi\right)+c_{2} \sinh \left(\frac{1}{2} \lambda_{1} \xi\right)}\right]}{\frac{2 b_{0}(m+n)}{ \pm n \sqrt{\frac{-b(m+2 n)}{a m k}}}-b_{1}\left[1-\frac{c_{1} \sinh \left(\frac{1}{2} \lambda_{1} \xi\right)+c_{2} \cosh \left(\frac{1}{2} \lambda_{1} \xi\right)}{c_{1} \cosh \left(\frac{1}{2} \lambda_{1} \xi\right)+c_{2} \sinh \left(\frac{1}{2} \lambda_{1} \xi\right)}\right]}\right\}^{\frac{1}{2 n}} \\
& \quad \times \exp \left(\mathrm{i}\left[k_{1} x-\left(a m k_{1}^{2}+\frac{b(m+2 n)}{4 k(m+n)^{2}}\right) t\right]\right) .
\end{aligned}
$$

If $c_{2}=0, c_{1} \neq 0$, then we have the dark soliton solution of Eq. (1.4):

$$
\begin{aligned}
& q(x, t)=\left\{\frac{\left[ \pm \frac{a m b_{0}}{2 n b} \sqrt{\frac{-b(m+2 n)}{a m k}}+\frac{b_{1}(m+2 n)}{2 k(m+n)}\right]\left[1-\tanh \left(\frac{1}{2} \sqrt{\frac{-n^{2} b(m+2 n)}{a m k(m+n)^{2}}} \xi\right)\right]}{\frac{2 b_{0}(m+n)}{ \pm n \sqrt{\frac{-b(m+2 n)}{a m k}}}-b_{1}\left[1-\tanh \left(\frac{1}{2} \sqrt{\frac{-n^{2} b(m+2 n)}{a m k(m+n)^{2}}} \xi\right)\right]}\right\}^{\frac{1}{2 n}} \\
& \times \exp \left(\mathrm{i}\left[k_{1} x-\left(a m k_{1}^{2}+\frac{b(m+2 n)}{4 k(m+n)^{2}}\right) t\right]\right) .
\end{aligned}
$$

If $c_{1}=0, c_{2} \neq 0$, we have the singular soliton solution of Eq. (1.4):

$$
\begin{aligned}
& q(x, t)=\left\{\frac{\left[ \pm \frac{a m b_{0}}{2 n b} \sqrt{\frac{-b(m+2 n)}{a m k}}+\frac{b_{1}(m+2 n)}{2 k(m+n)}\right]\left[1-\operatorname{coth}\left(\frac{1}{2} \sqrt{\frac{-n^{2} b(m+2 n)}{a m k(m+n)^{2}}} \xi\right)\right]}{\frac{2 b_{0}(m+n)}{ \pm n \sqrt{\frac{-b(m+2 n)}{a m k}}}-b_{1}\left[1-\operatorname{coth}\left(\frac{1}{2} \sqrt{\frac{-n^{2} b(m+2 n)}{a m k(m+n)^{2}}} \xi\right)\right]}\right\}^{\frac{1}{2 n}} \\
& \times \exp \left(\mathrm{i}\left[k_{1} x-\left(a m k_{1}^{2}+\frac{b(m+2 n)}{4 k(m+n)^{2}}\right) t\right]\right) .
\end{aligned}
$$


The constraint conditions for the existence of the soliton solutions (4.2.18)-(4.2.20) are $k<0$ and $a b>0$.

Set 4

$$
\begin{aligned}
& a_{0}=0, \quad a_{1}=0, \quad a_{2}=\mp \frac{a m b_{1}}{2 n b} \sqrt{\frac{-b(m+2 n)}{a m k}}, \quad b_{0}= \pm \frac{b_{1}}{4} \sqrt{\frac{-b n^{2}(m+2 n)}{a m k(m+n)^{2}}}, \quad \mu_{1}=0, \\
& \lambda_{1}= \pm \frac{1}{2} \sqrt{\frac{-b n^{2}(m+2 n)}{a m k(m+n)^{2}}}, \quad \omega=a m k_{1}^{2}+\frac{b(m+2 n)}{4 k(m+n)^{2}}, \quad b_{1}=b_{1},
\end{aligned}
$$

provided that $a b k<0$.

Substituting (4.2.21) into (4.2.2) yields

$$
v(\xi)=-\frac{a m}{2 n b} \sqrt{\frac{-b(m+2 n)}{a m k}}\left(\frac{G^{\prime}(\xi)}{G(\xi)}\right)^{2} /\left[\frac{1}{4} \sqrt{\frac{-b n^{2}(m+2 n)}{a m k(m+n)^{2}}} \pm\left(\frac{G^{\prime}(\xi)}{G(\xi)}\right)\right] .
$$

From (4.5),(4.1),(3.7) and using (4.2.22), we have the hyperbolic solution of Eq. (1.4):

$$
\begin{aligned}
& q(x, t)=\left\{\frac { ( m + 2 n ) } { 8 k ( m + n ) } \left[\frac{\left(1-\frac{c_{1} \sinh \left(\frac{1}{2} \lambda_{1} \xi\right)+c_{2} \cosh \left(\frac{1}{2} \lambda_{1} \xi\right)}{c_{1} \cosh \left(\frac{1}{2} \lambda_{1} \xi\right)+c_{2} \sinh \left(\frac{1}{2} \lambda_{1} \xi\right)}\right)^{2}}{\left.\left.\frac{c_{1} \sinh \left(\frac{1}{2} \lambda_{1} \xi\right)+c_{2} \cosh \left(\frac{1}{2} \lambda_{1} \xi\right)}{c_{1} \cosh \left(\frac{1}{2} \lambda_{1} \xi\right)+c_{2} \sinh \left(\frac{1}{2} \lambda_{1} \xi\right)}\right]\right\}^{\frac{1}{2 n}}}\right.\right. \\
& \quad \times \exp \left(\mathrm{i}\left[k_{1} x-\left(a m k_{1}^{2}+\frac{b(m+2 n)}{4 k(m+n)^{2}}\right) t\right]\right) .
\end{aligned}
$$

If $c_{2}=0, c_{1} \neq 0$, then we have the dark soliton solution of Eq. (1.4):

$$
\begin{aligned}
& q(x, t)=\left\{\frac{(m+2 n)}{8 k(m+n)}\left[\frac{\left(1-\tanh \left(\frac{1}{4} \sqrt{\frac{-n^{2} b(m+2 n)}{a m k(m+n)^{2}}} \xi\right)\right)^{2}}{\tanh \left(\frac{1}{4} \sqrt{\frac{-n^{2} b(m+2 n)}{a m k(m+n)^{2}}} \xi\right)}\right]\right\}^{\frac{1}{2 n}} \\
& \quad \times \exp \left(\mathrm{i}\left[k_{1} x-\left(a m k_{1}^{2}+\frac{b(m+2 n)}{4 k(m+n)^{2}}\right) t\right]\right) .
\end{aligned}
$$

If $c_{1}=0, c_{2} \neq 0$, then we have the singular soliton solution of Eq. (1.4):

Or

$$
\begin{aligned}
& q(x, t)=\left\{\frac{(m+2 n)}{8 k(m+n)}\left[\frac{\left(1-\operatorname{coth}\left(\frac{1}{4} \sqrt{\frac{-n^{2} b(m+2 n)}{a m k(m+n)^{2}}} \xi\right)\right)^{2}}{\operatorname{coth}\left(\frac{1}{4} \sqrt{\frac{-n^{2} b(m+2 n)}{a m k(m+n)^{2}}} \xi\right)}\right]\right\}^{\frac{1}{2 n}} \\
& \quad \times \exp \left(\mathrm{i}\left[k_{1} x-\left(a m k_{1}^{2}+\frac{b(m+2 n)}{4 k(m+n)^{2}}\right) t\right]\right) .
\end{aligned}
$$

$$
\begin{aligned}
& q(x, t)=\left[\frac{(m+2 n)}{8 k(m+n)}\left(\tanh \left(\frac{1}{4} \sqrt{\frac{-n^{2} b(m+2 n)}{a m k(m+n)^{2}} \xi}\right)+\operatorname{coth}\left(\frac{1}{4} \sqrt{\frac{-n^{2} b(m+2 n)}{a m k(m+n)^{2}}} \xi\right)-2\right)\right]^{\frac{1}{2 n}} \\
& \quad \times \exp \left(\mathrm{i}\left[k_{1} x-\left(a m k_{1}^{2}+\frac{b(m+2 n)}{4 k(m+n)^{2}}\right) t\right]\right) .
\end{aligned}
$$

The constraint conditions for the existence of the soliton solutions (4.2.23)-(4.2.26) are $k>0$ and $a b<0$.

Set 5

$$
\begin{aligned}
& a_{0}=a_{2} \mu_{1}, \quad a_{1}=\frac{-a_{2} n(m+2 n)}{k(m+n)} \sqrt{\frac{-k b}{a m(m+2 n)}}, \quad a_{2}=a_{2}, \quad b_{0}=0, \quad b_{1}=2 a_{2} n \sqrt{\frac{-k b}{a m(m+2 n)},} \\
& \lambda_{1}=\frac{n b}{a m(m+n) \sqrt{\frac{-k b}{a m(m+2 n)}}}, \quad \mu_{1}=\mu_{1}, \quad \omega=a m k_{1}^{2}+\frac{a m \mu_{1}}{n^{2}}+\frac{b(m+2 n)}{4 k(m+n)^{2}},
\end{aligned}
$$

provided that $a b k<0$. 
Substituting (4.2.27) into (4.2.2) yields

$$
v(\xi)=\frac{\mu_{1}-\frac{n(m+2 n)}{k(m+n)} \sqrt{\frac{-k b}{a m(m+2 n)}}\left(\frac{G^{\prime}(\xi)}{G(\xi)}\right)+\left(\frac{G^{\prime}(\xi)}{G(\xi)}\right)^{2}}{2 n \sqrt{\frac{-k b}{a m(m+2 n)}}\left(\frac{G^{\prime}(\xi)}{G(\xi)}\right)} .
$$

Now, we consider the following cases.

Case1: From (4.5),(4.1),(3.7) and using (4.2.28), we have the hyperbolic solution of Eq. (1.4):

$$
\begin{aligned}
& q(x, t)=\left\{\frac{\left(\frac{b n^{2}(m+2 n)}{4 a m k(m+n)^{2}}+\mu_{1}\right)\left[1-\left(\frac{c_{1} \sinh \left(\frac{1}{2} \sqrt{\lambda_{1}^{2}-4 \mu_{1}} \xi\right)+c_{2} \cosh \left(\frac{1}{2} \sqrt{\lambda_{1}^{2}-4 \mu_{1}} \xi\right)}{c_{1} \cosh \left(\frac{1}{2} \sqrt{\lambda_{1}^{2}-4 \mu_{1}} \xi\right)+c_{2} \sinh \left(\frac{1}{2} \sqrt{\lambda_{1}^{2}-4 \mu_{1}} \xi\right)}\right)^{2}\right]}{\frac{-b n^{2}}{a m(m+n)}+n \sqrt{k_{2}}\left(\frac{c_{1} \sinh \left(\frac{1}{2} \sqrt{\lambda_{1}^{2}-4 \mu_{1}} \xi\right)+c_{2} \cosh \left(\frac{1}{2} \sqrt{\lambda_{1}^{2}-4 \mu_{1}} \xi\right)}{c_{1} \cosh \left(\frac{1}{2} \sqrt{\lambda_{1}^{2}-4 \mu_{1}} \xi\right)+c_{2} \sinh \left(\frac{1}{2} \sqrt{\lambda_{1}^{2}-4 \mu_{1}} \xi\right)}\right\}^{\frac{1}{2 n}}}\right. \\
& \quad \times \exp \left(\mathrm{i}\left[k_{1} x-\left(a m k_{1}^{2}+\frac{a m \mu_{1}}{n^{2}}+\frac{b(m+2 n)}{4 k(m+n)^{2}}\right) t\right]\right),
\end{aligned}
$$

where $k_{2}=\frac{b^{2} n^{2}}{a^{2} m^{2}(m+n)^{2}}+\frac{4 k b \mu_{1}}{a m(m+2 n)}>0$ and $\lambda_{1}^{2}-4 \mu_{1}=\frac{-n^{2} b(m+2 n)}{a m k(m+n)^{2}}-4 \mu_{1}>0$.

If $c_{2}=0, c_{1} \neq 0$, then we have the solution of Eq. (1.4):

$$
\begin{aligned}
& q(x, t)=\left\{\frac{\left[\frac{b n^{2}(m+2 n)}{4 a m k(m+n)^{2}}+\mu_{1}\right] \operatorname{sech}^{2}\left(\frac{\xi}{2} \sqrt{\frac{-n^{2} b(m+2 n)}{a m k(m+n)^{2}}-4 \mu_{1}}\right)}{\frac{-b n^{2}}{a m(m+n)}+n \sqrt{k_{2}} \tanh \left(\frac{\xi}{2} \sqrt{\frac{-n^{2} b(m+2 n)}{a m k(m+n)^{2}}-4 \mu_{1}}\right)}\right\}^{\frac{1}{2 n}} \\
& \quad \times \exp \left(\mathrm{i}\left[k_{1} x-\left(a m k_{1}^{2}+\frac{a m \mu_{1}}{n^{2}}+\frac{b(m+2 n)}{4 k(m+n)^{2}}\right) t\right]\right) .
\end{aligned}
$$

If $c_{1}=0, c_{2} \neq 0$, then we have the solution of Eq. (1.4):

$$
\begin{aligned}
& q(x, t)=\left\{\frac{-\left[\frac{b n^{2}(m+2 n)}{4 a m k(m+n)^{2}}+\mu_{1}\right] \operatorname{csch}^{2}\left(\frac{\xi}{2} \sqrt{\frac{-n^{2} b(m+2 n)}{a m k(m+n)^{2}}-4 \mu_{1}}\right)}{\frac{-b n^{2}}{a m(m+n)}+n \sqrt{k_{2}} \operatorname{coth}\left(\frac{\xi}{2} \sqrt{\frac{-n^{2} b(m+2 n)}{a m k(m+n)^{2}}-4 \mu_{1}}\right)}\right\}^{\frac{1}{2 n}} \\
& \quad \times \exp \left(\mathrm{i}\left[k_{1} x-\left(a m k_{1}^{2}+\frac{a m \mu_{1}}{n^{2}}+\frac{b(m+2 n)}{4 k(m+n)^{2}}\right) t\right]\right) .
\end{aligned}
$$

The constraint conditions for the existence of the soliton solutions (4.2.30) and (4.2.31) are $k_{2}>0$ and $\frac{-n^{2} b(m+2 n)}{a m k(m+n)^{2}}-$ $4 \mu_{1}>0$.

Case 2: From (4.5),(4.1),(3.8) and using (4.2.28), we have the trigonometric periodic solution of Eq. (1.4):

$$
\begin{aligned}
& q(x, t)=\left\{\frac{\left[\frac{b n^{2}(m+2 n)}{4 a m k(m+n)^{2}}+\mu_{1}\right]\left[1+\left(\frac{c_{1} \cos \left(\frac{1}{2} \sqrt{4 \mu_{1}-\lambda_{1}^{2}} \xi\right)-c_{2} \sin \left(\frac{1}{2} \sqrt{4 \mu_{1}-\lambda_{1}^{2}} \xi\right)}{c_{1} \sin \left(\frac{1}{2} \sqrt{4 \mu_{1}-\lambda_{1}^{2}} \xi\right)+c_{2} \cos \left(\frac{1}{2} \sqrt{4 \mu_{1}-\lambda_{1}^{2}} \xi\right)}\right)^{2}\right]}{\frac{-b n^{2}}{a m(m+n)}+n \sqrt{-k_{2}}\left(\frac{c_{1} \cos \left(\frac{1}{2} \sqrt{4 \mu_{1}-\lambda_{1}^{2}} \xi\right)-c_{2} \sin \left(\frac{1}{2} \sqrt{4 \mu_{1}-\lambda_{1}^{2}} \xi\right)}{c_{1} \sin \left(\frac{1}{2} \sqrt{4 \mu_{1}-\lambda_{1}^{2}} \xi\right)+c_{2} \cos \left(\frac{1}{2} \sqrt{4 \mu_{1}-\lambda_{1}^{2}} \xi\right)}\right)^{\frac{1}{2 n}}}\right. \\
& \quad \times \exp \left(\mathrm{i}\left[k_{1} x-\left(a m k_{1}^{2}+\frac{a m \mu_{1}}{n^{2}}+\frac{b(m+2 n)}{4 k(m+n)^{2}}\right) t\right]\right),
\end{aligned}
$$

where $k_{2}=\frac{b^{2} n^{2}}{a^{2} m^{2}(m+n)^{2}}+\frac{4 k b \mu_{1}}{a m(m+2 n)}<0$ and $4 \mu_{1}-\lambda_{1}^{2}=\frac{n^{2} b(m+2 n)}{a m k(m+n)^{2}}+4 \mu_{1}>0$.

If $c_{1}=0, c_{2} \neq 0$, then Eq. (1.4) have the following periodic wave solution:

$$
\begin{aligned}
& q(x, t)=\left\{\frac{\left[\frac{b n^{2}(m+2 n)}{4 a m k(m+n)^{2}}+\mu_{1}\right] \sec ^{2}\left(\frac{\xi}{2} \sqrt{\frac{n^{2} b(m+2 n)}{a m k(m+n)^{2}}+4 \mu_{1}}\right)}{\frac{-b n^{2}}{a m(m+n)}-n \sqrt{-k_{2}} \tan \left(\frac{\xi}{2} \sqrt{\frac{n^{2} b(m+2 n)}{a m k(m+n)^{2}}+4 \mu_{1}}\right)}\right\}^{\frac{1}{2 n}} \\
& \quad \times \exp \left(\mathrm{i}\left[k_{1} x-\left(a m k_{1}^{2}+\frac{a m \mu_{1}}{n^{2}}+\frac{b(m+2 n)}{4 k(m+n)^{2}}\right) t\right]\right),
\end{aligned}
$$

while, if $c_{2}=0, c_{1} \neq 0$, then Eq. (1.4) have the following periodic wave solution: 


$$
\begin{gathered}
q(x, t)=\left\{\frac{\left[\frac{b n^{2}(m+2 n)}{4 a m k(m+n)^{2}}+\mu_{1}\right] \csc ^{2}\left(\frac{\xi}{2} \sqrt{\frac{n^{2} b(m+2 n)}{a m k(m+n)^{2}}+4 \mu_{1}}\right)}{\frac{-b n^{2}}{a m(m+n)}+n \sqrt{-k_{2}} \cot \left(\frac{\xi}{2} \sqrt{\frac{n^{2} b(m+2 n)}{a m k(m+n)^{2}}+4 \mu_{1}}\right)}\right\}^{\frac{1}{2 n}} \\
\quad \times \exp \left(\mathrm{i}\left[k_{1} x-\left(a m k_{1}^{2}+\frac{a m \mu_{1}}{n^{2}}+\frac{b(m+2 n)}{4 k(m+n)^{2}}\right) t\right]\right) .
\end{gathered}
$$

The constraint conditions for the existence of the periodic wave solutions (4.2.33) and $(4.2 .34)$ are $k_{2}<0$ and $\frac{n^{2} b(m+2 n)}{a m k(m+n)^{2}}+4 \mu_{1}>0$.

Case 3: If $\lambda_{1}^{2}-4 \mu_{1}=0$, that is to say $b=\frac{-4 \mu_{1} a m k(m+n)^{2}}{n^{2}(m+2 n)}$, then from (4.5),(4.1),(3.9) and using (4.2.28), we have the rational solution of Eq. (1.4):

$$
q(x, t)=\left\{\frac{(m+2 n)}{4 k(m+n)} \frac{c_{2}^{2}}{\left(c_{1}+c_{2} \xi\right)\left[\mu_{1}\left(c_{1}+c_{2} \xi\right)+c_{2} \sqrt{\mu_{1}}\right]}\right\}^{\frac{1}{2 n}} \exp \left(\mathrm{i}\left[k_{1} x-a m k_{1}^{2} t\right]\right),
$$

The constraint conditions for the existence of the rational solution (4.2.35) are $k>0$ and $\mu_{1}>0$.

Note that the solutions $(4.2 .13)-(4.2 .15),(4.2 .18)-(4.2 .20),(4.2 .23)-(4.2 .26)$, and (4.2.29)-(4.2.35) are new and not published elsewhere.

\section{Conclusion}

In this article, we apply two mathematical tools, namely the first integral method and the rational $\left(G^{\prime} / G\right)$ expansion method to find the exact traveling wave solutions, the optical bright-dark soliton solutions, some trigonometric function solutions and rational solutions of the nonlinear Biswas-Milovic equation with dual-power law nonlinearity (1.4). From our results, we conclude that the rational $\left(G^{\prime} / G\right)$-expansion method gives more solutions than the first integral method. On comparing our results obtained in this article using two different methods with the well-known results obtained in [66-69] using other different methods, we conclude that many results for Eq. (1.4) are new and not published elsewhere. Further, the different methods used in this paper are very powerful and effective techniques in finding the exact solutions and optical solitary wave solutions for a wide range of nonlinear problem. Finally, our results obtained in this article have been checked with the aid of the Maple by putting them back into the original Eq. (1.4).

\section{Acknowledgments}

The authors wish to thank the referee for his comments on this paper.

\section{References}

[1] M.J. Ablowitz, P.A. Clarkson, Solitons, Nonlinear Evolution Equations and Inverse Scattering Transform, Cambridge University Press, New York 1991.

[2] R. Hirota, Phys. Rev. Lett. 27, 1192 (1971).

[3] J. Weiss, M. Tabor, G. Carnevale, J. Math. Phys. 24, 522 (1983).

[4] N.A. Kudryashov, J. Appl. Math. Mech. 52, 361 (1988).

[5] N.A. Kudryashov, Phys. Lett. A 147, 287 (1990).

[6] N.A. Kudryashov, Phys. Lett. A 155, 269 (1991).

[7] M.R. Miura, Bäcklund Transformation, Springer, Berlin 1978.
[8] C. Rogers, W.F. Shadwick, Bäcklund Transformations and their Applications, Academic Press, New York 1982.

[9] J.H. He, X.H. Wu, Chaos Solitons Fract. 30, 700 (2006).

[10] S. Zhang, Chaos Solitons Fract. 38, 270 (2008).

[11] E.M.E. Zayed, A.-G. Al-Nowehy, Optik 127, 4970 (2016).

[12] E.G. Fan, Phys. Lett. A 277, 212 (2000).

[13] S. Zhang, T.C. Xia, Appl. Math. E-Notes 8, 58 (2008).

[14] Y. Chen, Q. Wang, Chaos Solitons Fract. 24, 745 (2005).

[15] S. Liu, Z. Fu, S. Liu, Q. Zhao, Phys. Lett. A 289, 69 (2001).

[16] D. Lu, Chaos Solitons Fract. 24, 1373 (2005).

[17] Q. Zhou, Q. Zhu, Y. Liu, H. Yu, P. Yao, A. Biswas, Laser Phys. 25, 015402 (2015).

[18] Q. Zhou, Q. Zhu, Y. Liu, H. Yu, C. Wei, P. Yao, A.H. Bhrawy, A. Biswas, Laser Phys. 25, 025402 (2015).

[19] E.M.E. Zayed, J. Phys. A Math. Theor. 42, 195202 (2009).

[20] M.L. Wang, X. Li, J. Zhang, Phys. Lett. A 372, 417 (2008).

[21] S. Zhang, J.L. Tong, W. Wang, Phys. Lett. A 372, 2254 (2008).

[22] E.M.E. Zayed, K.A. Gepreel, J. Math. Phys. 50, 013502 (2009).

[23] N.A. Kudryashov, Appl. Math. Comput. 217, 1755 (2010).

[24] E.M.E. Zayed, J. Appl. Math. Inform. 28, 383 (2010).

[25] M. Mirzzadeh, M. Eslami, Q. Zhou, M.F. Mahmood, E. Zerrad, A. Biswas, M. Belic, J. Nonlin. Opt. Phys. Mater. 24, 1550017 (2015).

[26] S. Zhang, Y.N. Sun, J.M. Ba, L. Dong, Z. Naturforsch. 66a, 33 (2011).

[27] L.X. Li, Q.E. Li, L.M. Wang, Appl. Math. J. Chin. Uni. 25, 454 (2010). 
[28] E.M.E. Zayed, M.A.M. Abdelaziz, Math. Prob. Eng. 2012, 725061 (2012).

[29] E.M.E. Zayed, K.A.E. Alurrfi, Math. Prob. Eng. 2014, 521712 (2014).

[30] E.M.E. Zayed, K.A.E. Alurrfi, Math. Prob. Eng. 2014, 746538 (2014).

[31] A.J.M. Jawad, M.D. Petkovic, A. Biswas, Appl. Math. Comput. 217, 869 (2010).

[32] E.M.E. Zayed, Appl. Math. Comput. 218, 3962 (2011).

[33] E.M.E. Zayed, A.-G. Al-Nowehy, Z. Naturforsch. 71a, 103 (2016)

[34] W.X. Ma, T. Huang, Y. Zhang, Phys. Scr. 82, 065003 (2010).

[35] E.M.E. Zayed, A.-G. Al-Nowehy, Z. Naturforsch. 70a, 775 (2015)

[36] W.X. Ma, J.H. Lee, Chaos Solitons Fract. 42, 1356 (2009).

[37] A.M. Yang, X.J. Yang, Z.B. Li, Abstr. Appl. Anal. 2013, 351057 (2013).

[38] R.M. El-Shiekh, A.-G. Al-Nowehy, Z. Naturforsch. 68a, 255 (2013).

[39] G.M. Moatimid, R.M. El-Shiekh, A.-G. Al-Nowehy, Nonlin. Sci. Lett. A 4, 1 (2013).

[40] M. Ekici, M. Mirzazadeh, M. Eslami, Q. Zhou, S.P. Moshokoa, A. Biswas, M. Belic, Optik 127, 10659 (2016).

[41] E.M.E. Zayed, Y.A. Amer, R.M.A. Shohib, Int. J. Phys. Sci. 8, 1246 (2013).

[42] S.D. Zhu, Chaos Solitons Fract. 37, 1335 (2008).

[43] A. Biswas, D. Milovic, M. Edwards, Mathematical Theory of Dispersion-Managed Optical Solitons, Springer-Verlag, New York 2010.

[44] A.K. Sarma, M. Saha, A. Biswas, J. Infrared Millim. THz Waves 31, 1048 (2010).

[45] A. Biswas, Commun. Nonlin. Sci. Numer. Simul. 15 $2744(2010)$

[46] A.H. Bhrawy, M.A. Abdelkawy, A. Biswas, Indian J. Phys. 87, 1125 (2013).

[47] Q. Zhou, Q. Zhu, M. Savescu, A. Bhrawy, A. Biswas, Proc. Roman. Acad. Ser. A 16, 152 (2015).

[48] A.C. Cevikela, E. Aksoy, Ö. Günerb, A. Bekir, Int. J. Nonlin. Sci. 16, 195 (2013).

[49] A.H. Bhrawy, M.A. Abdelkawy, A. Biswas, Optik 125, 1537 (2014).

[50] H. Triki, F. Azzouzi, A. Biswas, S.P. Moshokoa, M. Belic, Optik 128, 172 (2017).

[51] M. Savescu, Q. Zhou, L. Moraru, A. Biswas, S.P. Moshokoa, M. Belic, Optik 127, 8995 (2016).

[52] A.K. Sarma, A. Biswas, Opt. Appl. 41, 205 (2014).
[53] A.A. Alshaery, A.H. Bhrawy, E.M. Hilal, A. Biswas, J. Electromagn. Waves Appl. 28, 275 (2014).

[54] M. Savescu, S. Johnson, A.H. Kara, S.H. Crutcher, R. Kohl, A. Biswas, J. Electromagn. Waves Appl. 28, $242(2014)$.

[55] H. Triki, A. Biswas, D. Milovic, M. Belic, Acto Phys. Pol. A 130, 718 (2016).

[56] H. Triki, A. Biswas, D. Milovic, M. Belic, Opt. Commun. 366, 362 (2016).

[57] H. Triki, A. Biswas, S.P. Moshokoa, M. Belic, Optik 128, 71 (2017).

[58] Q. Zhou, Y. Zhong, M. Mirzazadeh, A.H. Bhrawy, E. Zerrad, A. Biswas, Waves Rand. Compl. Media 26, 204 (2016).

[59] S. Jana, Shivani, G.S. Parmar, B. Kaur, Q. Zhou, A. Biswas, M. Belic, Optoelectron. Adv. Mater. Rapid Commun. 10, 143 (2016).

[60] B. Kaur, S. Jana, Q. Zhou, A. Biswas, M. Belic, J. Optoelectron. Adv. Mater. 18, 428 (2016).

[61] M. Mirzazadeh, M. Ekici, A. Sonomezoglu, Q. Zhou, H. Triki, S. P. Moshokoa, A. Biswas, M. Belic, Optik 127, 11311 (2016).

[62] A.H. Kara, A. Biswas, M. Belic, Optik 127, 11662 (2016).

[63] S. Kumar, Q. Zhou, A.H. Bhrawy, E. Zerrad, A. Biswas, M. Belic, Roman. Rep. Phys. 68, 341 (2016).

[64] G. Ebadi, A. Mojavir, J.V. Guzman, K.R. Khan, M.F. Mahmood, L. Moraru, A. Biswas, M. Belic, Optoelectron. Adv. Mater. Rapid Commun. 8, 828 (2014).

[65] H. Triki, A. Biswas, S.P. Moshokoa, M. Belic, Optik 128, 63 (2017).

[66] A. Biswas, D. Milovic, Commun. Nonlin. Sci. Num. Simul. 15, 1473 (2010).

[67] E.M.E. Zayed, K.A.E. Alurrfi, J. Adv. Phys. 11, 3001 (2015).

[68] E.M.E. Zayed, A.-G. Al-Nowehy, Open Phys. 14, 129 (2016).

[69] M. Mirzazadeh, M. Eslami, A.H. Arnous, Eur. Phys. J. Plus 130, 1 (2015).

[70] A. Biswas, Quant. Phys. Lett. 1, 79 (2012).

[71] S.Z. Feng, J. Phys. A Math. Gen. 35, 343 (2002).

[72] B. Zheng, WSEAS Trans. Math. 10, 249 (2011).

[73] E.M.E. Zayed, Y.A. Amer, Comput. Math. Model. 27, 80 (2016)

[74] E.M.E. Zayed, A.-G. Al-Nowehy, J. Part. Diff. Eq. 29, 218 (2016).

[75] W.X. Ma, B. Fuchssteiner, Int. J. Nonlin. Mech. 31, 329 (1996). 